\title{
Error Estimation Scheme Using AMI Code for Detect-and-Forward Multi-hop Communications
}

\author{
Kotoko Yamada and Kouji Ohuchi
}

\begin{abstract}
DetF (Detect-and-Forward) is one of the relaying schemes for multi-hop communications. In DetF, each relay node determines the data bit in the packet by hard-decision and only the destination node decodes and corrects the packet. If each relay node can observe how many errors are accumulated in the packet it relays, it can notice a good opportunity for decoding and correcting the accumulated errors in the packet. For DetF with error detection, we need the error detection scheme which can detect not only the existence of errors but the amount of errors. This paper focuses on a modified TPSK (ternary phase shift keying) using AMI (alternate mark inversion) code which can estimate the amount of errors easily. We compare this error detection scheme with BPSK (binary phase shift keying) using parity check code. This paper evaluates error estimation performance of these error detection schemes in multi-hop communications under fading environments. As a result, the modified TPSK with phases 0,135 and $-135 \mathrm{deg}$ is the most proper to an error detection scheme for DetF.
\end{abstract}

Index Terms-Multi-hop network, parity check code, AMI code, error estimation.

\section{INTRODUCTION}

In wireless ad-hoc networks, multi-hop networks are paid attention. It enables us to telecommunicate and construct quick and easy networking without cabling. However, the mean transmission time increases and the bit error rate deteriorates with an increase of the relay nodes. To achieve efficient multi-hop networks, various relaying schemes have been studied.

AF (amplify-and-forward) and DF (decode-and-forward) are one of the typical relaying schemes [1], [2]. AF relays a packet to the following node after performing only amplification of a packet signal in each relay node [3]. Although it has advantages that a system configuration is simple and transmission delay is small, there is a fault that errors are accumulated for every relay. In DF, an FEC (Forward Error Correction) technique is introduced. Each node decodes and corrects the received packet and relays it after re-encoding. In this scheme, since an error is not accumulated, the quality of the relayed data is maintained. However, transmission delay becomes large and loads of relay nodes increase. As a compromise between AF and DF, there is a relaying scheme called DetF (detect-and-forward). Although DetF also uses an FEC technique, only the destination node decodes and corrects the packet. Each relay

Manuscript received October 15, 2014; revised March 19, 2015

The authors are with the Graduate School of Engineering Shizuoka University, Johoku3-5-1, Naka, Hamamatsu, Shizuoka, 432-8561, Japan (e-mail: f0330144@ipc.shizuoka.ac.jp,dkoouti@ipc.shizuoka.ac.jp). node does not decode the packet but only determines the data bit in the packet by hard-decision. Thus, the accumulated errors in DetF are less than that in AF, but more than that in DF. When the destination node fails in the correction, it requests retransmission to the preceding node. Retransmission needs some costs such as further transmission delay and additional power consumption. If each relay node can observe how many errors are accumulated in the packet it relays, the relay node can notice a good opportunity for decoding and correcting the accumulated errors in the packet. Thus, when we use an error detection scheme to DetF, on-demand error correction can be achieved and it contributes toward reducing the retransmission costs.

We need the error detection schemes which can easily estimate the amount of errors contained in a packet. A simple attempt to estimate the amount of errors in a packet was introduced by Hatano et al. [2]. They used PC (parity check) code as an error detection code. Although PC code can detect errors easily, it needs extra bits for the parity. We pay attention to AMI (alternate mark inversion) code which can detect errors without extra bits. However, a normal AMI code is inferior in BER (bit error rate) to PC code. We proposed TPSK (ternary phase shift keying) using AMI code and it improved BER [4]. We also showed that the error estimation performance of TPSK using AMI code were superior to that of BPSK (binary phase shift keying) using PC code under Rayleigh fading environments.

To improve error estimation performance, we modify the TPSK constellation. In this study, we examine the BER and error estimation performance of the modified TPSK using AMI code and BPSK using PC code. We analyze these performances under Rayleigh and Rice fading environments by simulations [5].

\section{RELAYING SCHEMES}

\section{A. AF Scheme and DF Scheme [1]}

There are AF and DF schemes as a relaying scheme for multi-hop communications. In AF, each relay node amplifies a packet signal and relays it to the following node. It has advantages that the system configuration is simple and transmission delay is small. However, BER at destination node deteriorates since errors are accumulated for every relay and noise is also amplified in each relay node.

In $\mathrm{DF}$, each relay node decodes a received packet and relays it after re-encoding and re-modulating. We can reduce noise in the packet since each node decodes and noise is not amplified. However, there is fault that transmission delay becomes large and loads of relay nodes increase. If we use 
mobile terminals as the relay node, this increase of loads causes serious problems.

\section{B. DetF Scheme}

In DetF, each relay node transmits a packet to the following node after determining the data bit in the packet by hard-decision. Only the destination node decodes the packet. Loads of relay nodes in DetF are smaller than that in DF. However, performance deteriorates since soft-information of the data bit in the packet is wasted by hard-decision. Thus, in the destination node, DetF is more likely to fail in the correction than DF does. When the destination node fails in the correction, it requests retransmission to the preceding node. Retransmission needs some costs such as further transmission delay and additional power consumption.

To improve DetF scheme, the relaying scheme in which each relay node detects errors in the packet is useful. For example, we can decode the packet only when each relay node detects the existence of errors by using an error detection code, e.g. CRC (Cyclic Redundancy Check) code. If we use this scheme, the quality of the relayed data is maintained. However, if errors are few, but not zero, the relay node need not decode the packet since the destination node may succeed in the correction. Thus, we need the error detection code which can detect not the existence of errors but the amount of errors to reduce the unnecessary decoding. CRC code is not proper for this error detection scheme since it can detect only the existence of errors. The relay node can notice a good opportunity for decoding and correcting the accumulated errors in the packet by using a proper error detection scheme to DetF.

Hatano et al. [2] used PC code as an error detection code which can estimate the amount of errors in a packet. We use AMI code. In this study, we compare the performance of the modified TPSK using AMI code with BPSK using PC code.

\section{ERROR DETECTION CODE}

\section{A. BPSK Using Parity Check Code}

Parity check code is a simple error detection code which can estimate the amount of errors easily. However, it needs extra bits for the parity. We explain the error detection principle of even PC code in Fig. 1. In PC code, we call a period between the inserted PC bits a block. In this study, PC code is modulated by BPSK.

In a relay node, the number of +1 's in a block is counted. If we use even PC code, we can detect the existence of an error in the block when the number of +1 's is odd. We show this situation in Fig. 1(a). In this example, we mistake signal -1 for +1 . Thus, we can find an error in this block because the number of +1 's is odd. However, if two or more errors occur in a block, we cannot detect them correctly. For example, if two errors occur in a block as shown in Fig. 1(b), the number of +1 's is even. In this case, although errors occur, we cannot find these errors. If three errors occur in a block, we can detect the existence of an error because the number of +1 's is odd.

However, PC code cannot detect the amount of errors in a block. Thus, we suppose an error exists in a block even if three errors occur.

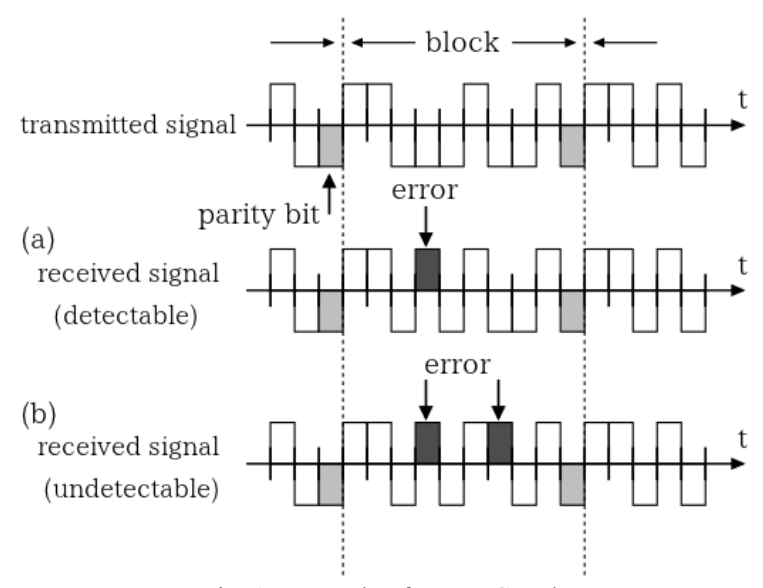

Fig. 1. Example of even PC code.

As can be seen in this example, PC code cannot detect the existence of errors when even errors occur in a block and cannot always detect errors correctly when three or more odd number of errors occurs in a block.

\section{B. TPSK Using AMI Code}

We explain the error detection principle of AMI code in Fig. 2. In a normal AMI code, there are three signal states: 0, +1 , and -1 . When we transmit binary data " 0,1 " by the normal AMI code, we use signal 0 for data " 0 ", and signal +1 or -1 alternatively for data " 1 ". Thus, if an error occurs and the resulting signals do not have +1 and -1 alternatively, we can detect the existence of an error. However, we cannot notice which data bit is erroneous. For example, if errors occur as shown in Fig. 2(a), we can detect them since the same polarity signals are successive. However, if errors occur near by each other as shown in Fig. 2(b), we cannot detect them. Thus, AMI code cannot detect all errors in a packet, too. Signal points of a normal AMI code and the modified TPSK using AMI code are shown in Fig. 3(a) and Fig. 3(b), respectively. The average signal powers of these constellations are equivalent. The minimum distance between adjacent signal points of the normal AMI code which has a constellation in Fig. 3(a) and BPSK using PC code are $\sqrt{2}$ and 2, respectively. Thus, the normal AMI code is inferior in BER to BPSK using PC code because its minimum distance between adjacent signal points is shorter than BPSK using PC code's one. To improve the BER degradation, we use the TPSK constellation as shown in Fig. 3(b). The minimum distance between adjacent signal points of TPSK using AMI code with $\theta_{X}=120$ deg is $\sqrt{3}$. Thus, BER of TPSK using AMI code is superior to that of the normal AMI code. Besides, the BER improves as $\theta_{X}$ in Fig. 3(b) increases. We call TPSK with $\theta_{X} \neq 120 \mathrm{deg}$ the modified TPSK.

In the modified TPSK using AMI code, we should note that there is a case in which an error occurs and we mistake signal $+1(-1)$ for $-1(+1)$. This error can be also counted in the error detection procedure of AMI code. However this case causes no bit error because both signals +1 and -1 represent data bit 1 . As the result, this case will cause excessive detection. The excessive detection causes deterioration of error estimation. For example, when we mistake signal -1 for +1 , we detect two errors by the above detection rule. However, we need not detect the errors because it is not bit error. Thus, to reduce the 
excessive detection, if the same polarity signals appear an odd number of times consecutively in the received signal, we do not count them in the number of detected errors as shown Fig. 4. However, we cannot always relieve all excessive detection and the excessive detection increase as $\theta_{X}$ increases.
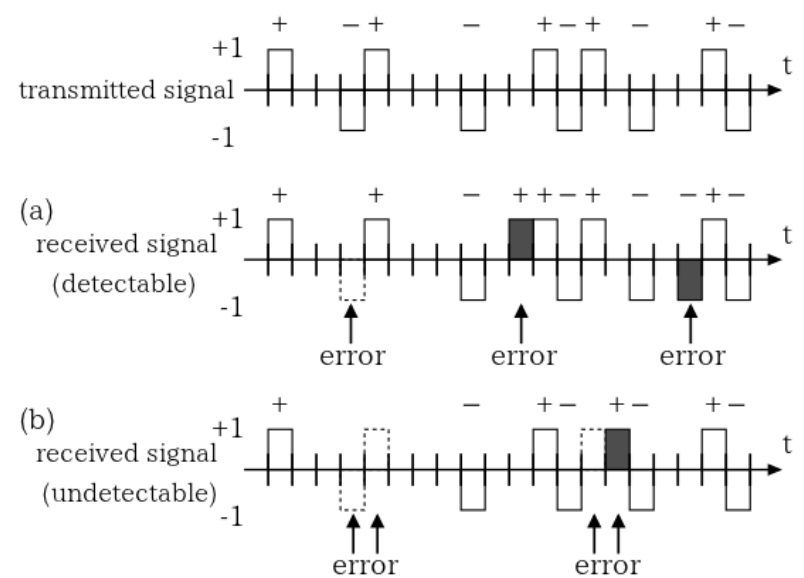

Fig. 2. Example of AMI code.
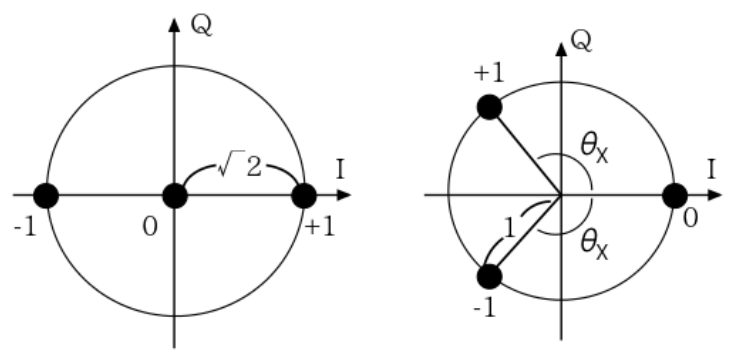

(a) AMI (normal)

(b) modified TPSK using AMI

Fig. 3. Constellations of a normal AMI code and modified TPSK using AMI code.

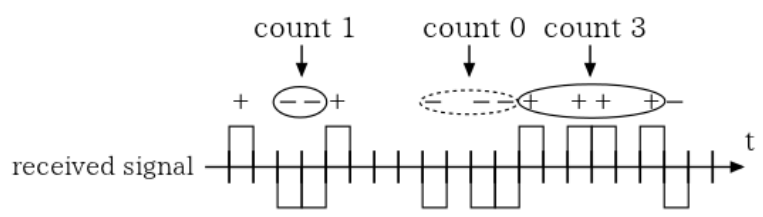

Fig. 4. Error detection method of modified TPSK using AMI code.

\section{PERFORMANCE ANALYSIS}

In this section, we investigate error estimation performance of BPSK using PC code and TPSK using AMI code in single-hop and multi-hop communications under Rayleigh and Rice fading environments. In this study, the packet size is 1000 bit. BPSK using PC code has 10 percent parity bits. The Ricean $K$-factor is 1.0 .

\section{A. Analysis in Single-Hop}

In this subsection, we examine the BER and error estimation performance when the source node transmits a packet to the destination node in single-hop. We show the actual BER's and the estimated BER's of BPSK using PC code and TPSK using AMI with $\theta_{X}=120 \mathrm{deg}$ under Rayleigh fading environments obtained by simulations in Fig. 5. These BER's are the results before performing the error correction by FEC. The estimated BER means the proportion of the detected errors among the transmitted data bits. Thus, we detect errors correctly when there is no difference between the actual BER and the estimated BER.

We can observe that these modulation systems have a comparable BER owing to the influence of Rayleigh fading. Besides, the difference between the actual BER and the estimated BER of the modified TPSK using AMI code is smaller than that of BPSK using PC code. Thus, we find that the error detection performance of TPSK using AMI code is better than that of BPSK using PC code.

We show actual BER's of the modified TPSK using AMI code with $\theta_{X}=105,120,135$ and 150 deg in Fig. 6 . As can be seen in Fig. 6, the BER improves as $\theta_{X}$ increases. The BER of the modified TPSK using AMI code with $\theta_{X}=120 \mathrm{deg}$ or more is almost equivalent or superior to that of BPSK using PC code.

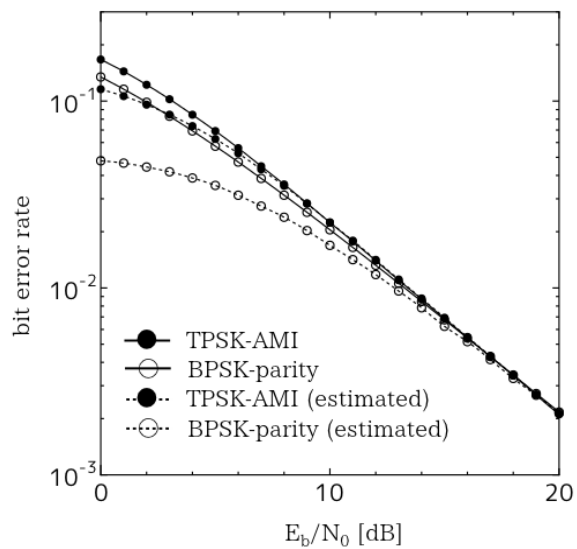

Fig. 5. Actual BER's and estimated BER's of each error detection scheme in single-hop (Rayleigh fading).

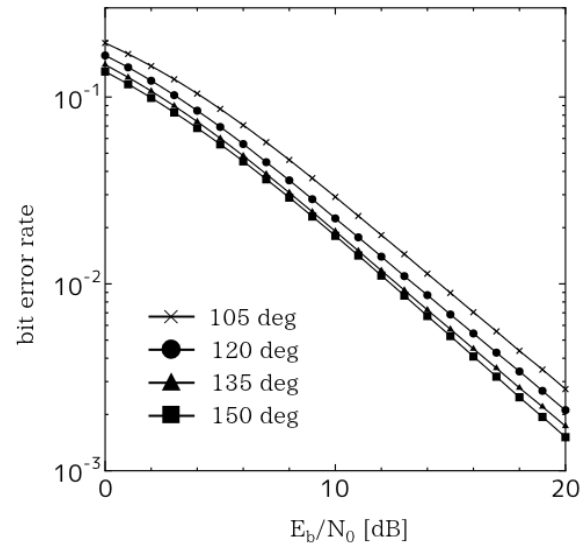

Fig. 6. BER's of the modified TPSK using AMI code (Rayleigh fading).

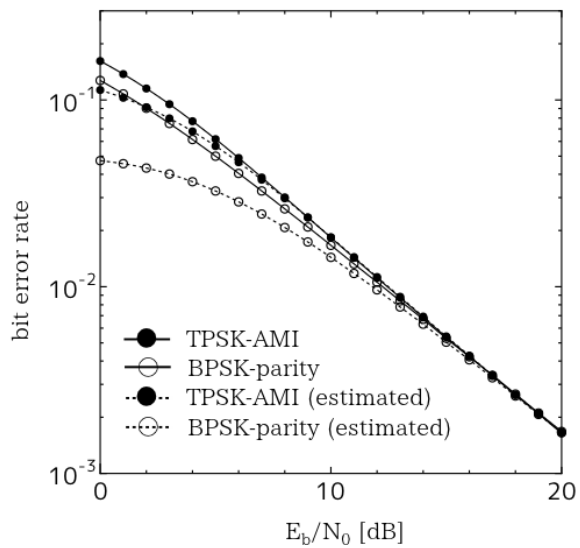

Fig. 7. Actual BER's and estimated BER's of each error detection scheme in single-hop (Rice fading). 


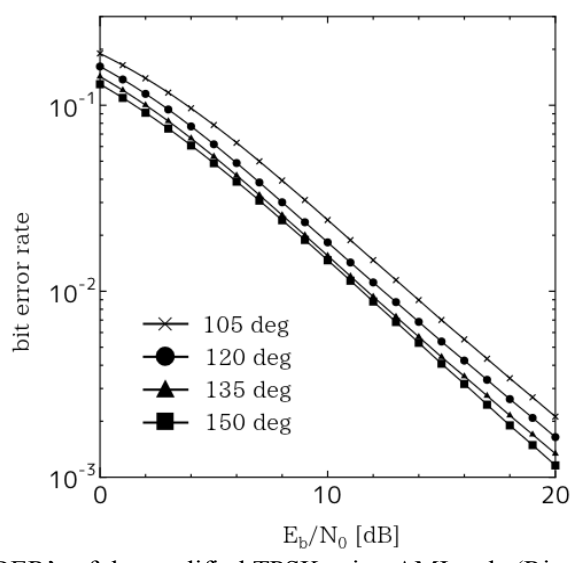

Fig. 8. BER's of the modified TPSK using AMI code (Rice fading).

Similarly, we show performance under Rice fading environments. Fig. 7 shows the actual BER's and the estimated BER's of each error detection scheme under Rice fading environments obtained by simulations. Fig. 8 shows actual BER's of the modified TPSK using AMI code with $\theta_{X}=105,120,135$ and $150 \mathrm{deg}$. We find that these results are similar to the performance under Rayleigh fading environments.

\section{B. Analysis in Multi-hop}

In previous subsection, we analyzed in single-hop. In this subsection, we analyze in multi-hop because we intend the error detection scheme to be used with DetF.

The estimated BER discords with the actual BER as shown in previous subsection. Thus, we revise the estimated BER by table mapping in order to improve accuracy of the error estimation. We revise the estimated BER by the following procedure; we make a table which describes the average estimated BER's and their corresponding actual BER's. Each node calculates the estimated BER from the amount of errors in the received packet and chooses the BER which corresponds to the estimated BER. We call this BER "revised BER". In brief, each node revises BER from the dotted line (the estimated BER) to the solid line (the actual BER) in Figs. 5 and 7 . We revise the estimated BER of both the modified TPSK using AMI code and BPSK using PC code. We show the correspondences between the actual BER and the estimated BER of the modified TPSK using AMI code with $\theta_{X}=120 \mathrm{deg}$ in Fig. 9 and Fig. 10. This correspondence is independent from the environment of channel. Thus, we can always use the same table.

When a relay node detects errors, the node decides whether it decodes the packet or not from the revised BER. If the node decides that the packet does not need to be decoded, it relays the packet to the following node. However, both of the error detection schemes mentioned above cannot detect all errors. We assume that many errors are accumulated but the relay node cannot detect the errors enough. In this case, it is possible that the node does not decode the packet although the packet needs to be decoded. We call this situation "overlooking". We also call the node that the overlooking occurs "overlooking node". Once an overlooking occurs, even if a following node can notice that the packet already accumulates many errors, it may fail in the correction. Thus the retransmission request increases as the overlooking increases. By using an error detection scheme, each relay node detects errors and obtains the revised BER. We assume that the relay node decides to start decoding when the revised BER exceeds a threshold value. The overlooking occurs when the actual BER exceeds a threshold value but the revised BER does not exceed the threshold value. This threshold value varies depending on the ability of FEC that we use in DetF [6]. Therefore, this value can be set arbitrarily. In this study, the threshold value is set at 0.05 . Besides the overlooking, we have to consider the number of hops which it takes until the relay node decides to start decoding the packet.

We show the overlooking rates of each error detection scheme under Rayleigh fading environments in Fig. 11. We also show the average number of hops of each error detection scheme in Fig. 12. As can be seen in Fig. 11, the overlooking rate of the modified TPSK using AMI code is lower than that of BPSK using PC code. We can also find that the overlooking rate decrease as $\theta_{X}$ increases. However, the average number of hops is maximum when $\theta_{X}$ is $135 \mathrm{deg}$. When $\theta_{X}$ is more than $135 \mathrm{deg}$, the average number of hops decreases as $\theta_{X}$ increases. This is caused by the excessive detection. Fig. 13 and Fig. 14 show the average BER and the average revised BER of each number of hops under Rayleigh fading environments. Fig. 13 and Fig. 14 show the results when $\theta_{X}$ is $120 \mathrm{deg}$ and $150 \mathrm{deg}$, respectively. As can be seen in these results, the difference between the average actual BER and the average revised BER becomes large as the number of hops increases. These figures show that the excessive detection occurs. The difference for $\theta_{X}=150 \mathrm{deg}$ is larger than the difference for $\theta_{X}=120 \mathrm{deg}$. Thus, the excessive detection increases as $\theta_{X}$ increases. Therefore, when $\theta_{X}$ is more than $135 \mathrm{deg}$, both the overlooking rate and the average number of hops decrease owing to the increase in the excessive detection. This causes the unnecessary error correction and costs. Thus, the modified TPSK using AMI code with $\theta_{X}=135 \mathrm{deg}$ is most proper for an error detection scheme of DetF because it can relay the most and its overlooking rate is relatively low.

If the overlooking occurs, we should also know how many overlooking nodes exist. We show the average number of overlooking nodes under Rayleigh fading environments in Fig. 15. In the modified TPSK using AMI code, if a node fails in the correction, the node requests retransmission to the second preceding node since the number of node that overlooking occur is about 1 . In BPSK using PC code, the node requests retransmission to the third preceding node since the number of node that overlooking occur is about 2 . Thus, the number of hops to retransmission in BPSK using PC code is more than that in the modified TPSK using AMI code. Therefore, the modified TPSK using AMI code is superior to BPSK using PC code since it is preferred that the number of hops is small. In the modified TPSK using AMI code, the average number of overlooking nodes is maximum when $\theta_{X}$ is $135 \mathrm{deg}$ since the average number of hop is maximum when $\theta_{X}$ is $135 \mathrm{deg}$. However, it is not a big disadvantage since the differences between this angle and other angles are small.

Similarly, we show the overlooking rate and the average 
number of hops under Rice fading environments in Fig. 16. In Fig. 16, histograms show an overlooking rate and line graph shows the average number of hops of each error detection scheme. In Rice fading environments, the modified TPSK using AMI code with $\theta_{X}=135 \mathrm{deg}$ is most proper, too.

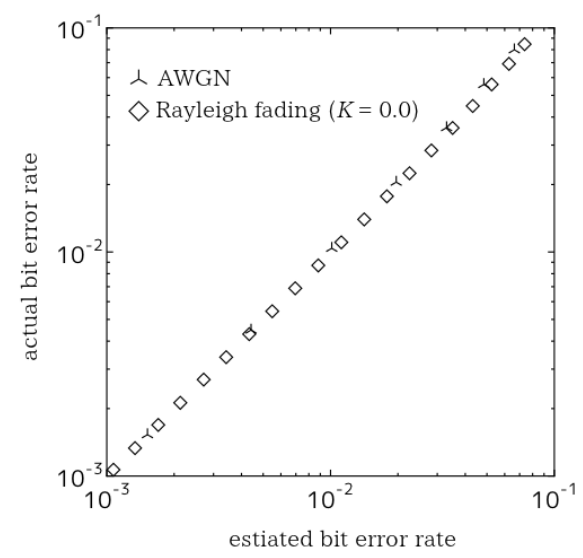

Fig. 9. Actual BER and estimated BER under AWGN and Rayleigh fading environments.

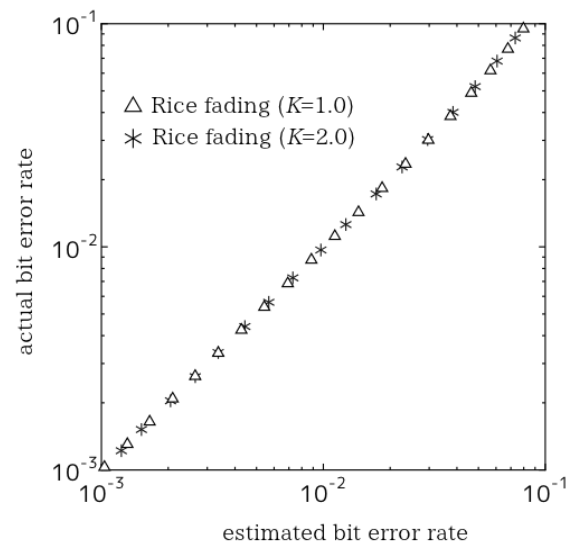

Fig. 10. Actual BER and estimated BER under Rice fading environments.

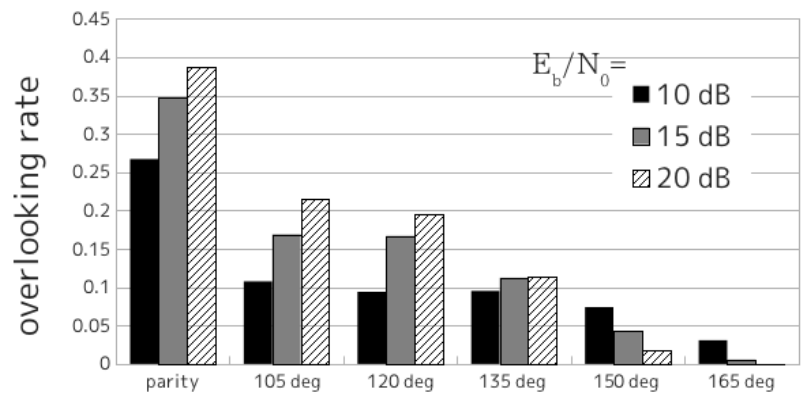

error detection scheme

Fig. 11. Overlooking rates of each error detection scheme (Rayleigh fading).

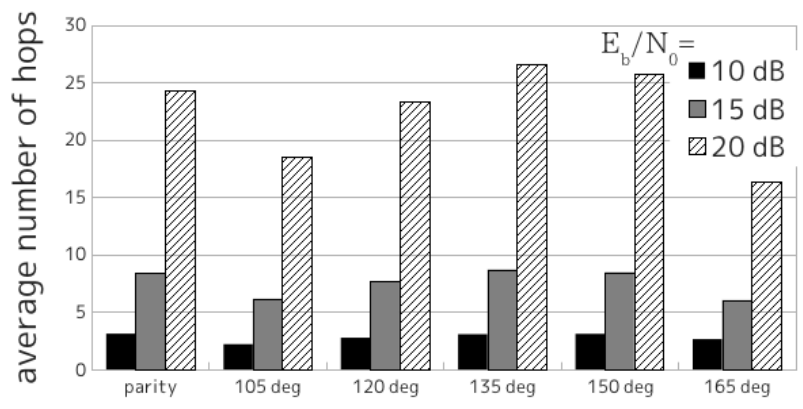

error detection scheme

Fig. 12. Average number of hops of each error detection scheme (Rayleigh fading).

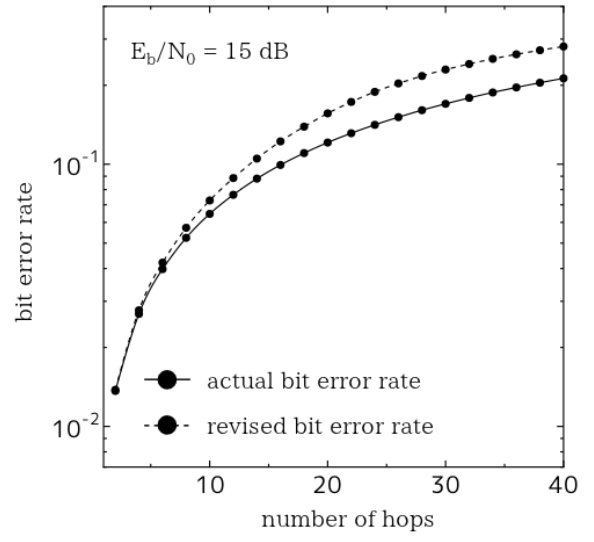

Fig. 13. Average actual BER and average revised BER of the modified TPSK using AMI code with $\theta_{X}=120$ deg (Rayleigh fading).

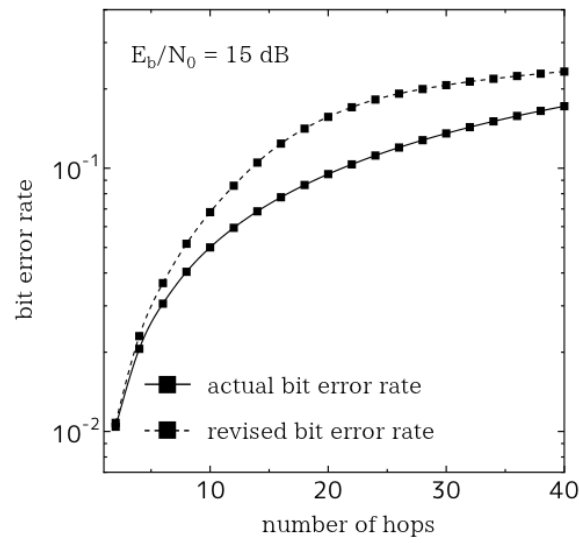

Fig. 14. Average actual BER and average revised BER of the modified TPSK using AMI code with $\theta_{X}=150 \mathrm{deg}$ (Rayleigh fading).

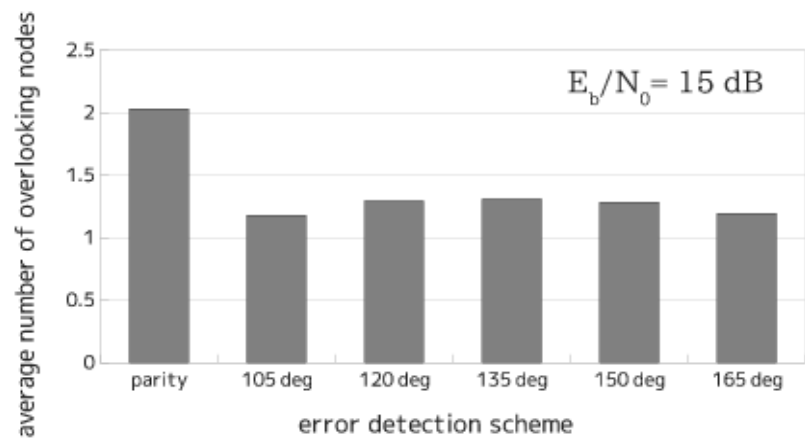

Fig. 15. Average number of overlooking nodes.

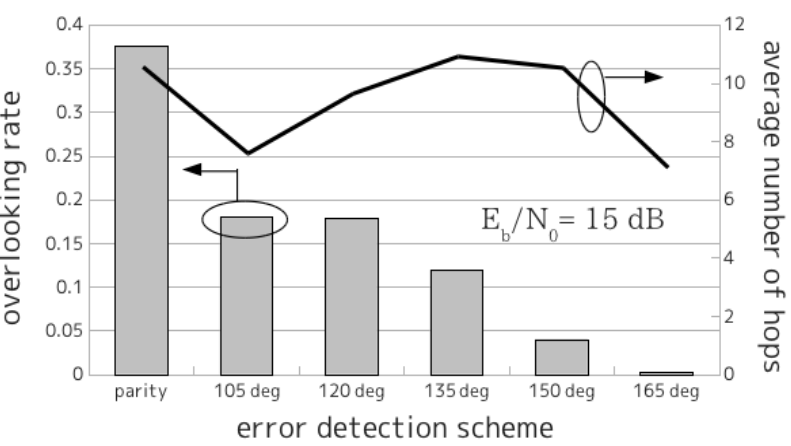

Fig. 16. Overlooking rates and average number of hops of each error detection scheme (Rice fading).

\section{CONCLUSION}

In DetF multi-hop communications, we investigated the BER and the error estimation performance of error detection 
schemes under fading environments. We focused on the modified TPSK using AMI code as a modulation system and compared with BPSK using PC code. In this paper, we showed the BER and the error estimation performance of each error detection scheme in single-hop and multi-hop.

The BER of the modified TPSK using AMI code with $\theta_{X}=120 \mathrm{deg}$ or more is almost equivalent or superior to that of BPSK using PC code. Error estimation performance of the modified TPSK using AMI code is superior to that of BPSK using PC code. In the modified TPSK using AMI code, the overlooking rate decreases as $\theta_{X}$ increases and the average number of hops is maximum when $\theta_{X}$ is $135 \mathrm{deg}$. Consequently, we can say that the modified TPSK using AMI code with $\theta_{X}=135 \mathrm{deg}$ is a most useful modulation system in DetF.

\section{REFERENCES}

[1] A. Nosratinia, T. E. Hunter, and A. Hedayat, "Cooperative communication in wireless networks," IEEE Communications Magazine, vol. 42, issue 10, pp. 74-80, October 2004.

[2] T. Hatano, T. Wada, and K. Ishibashi, "A study on new Hybrid ARQ scheme based on bit-by-bit likelihood update using packet combining (in Japanese)," IEICE Technical Report, RCS2010-64, pp. 95-99, July 2010.

[3] P. Popovski and H. Yomo, "Wireless network coding by amplify-and-forward for Bi-directional traffic flows," IEEE Communications Letters, vol. 11, no. 1, pp. 16-18, 2007.
[4] K. Yamada and K. Ohuchi, "Error detection performance of TPSK using AMI code in multi-hop communications under Rayleigh fading environments," IEICE Transactions on Fundamentals of Electronics, Communications and Computer Sciences, vol. E97-A, no. 12, December 2014.

[5] J. G. Proakis and M. Salehi, Digital Communications, $5^{\text {th }}$ ed. New York: McGraw-Hill, 2008, pp. 846-848.

[6] C. Berrow, A. Glavieux, and Thitimajshima, "Near Shannon limit error-correcting coding and decoding: Turbo-Codes (1)," in Proc. ICC, May 1993, pp. 1064-1070.

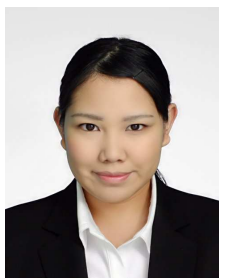

Kotoko Yamada was born in Shizuoka, Japan, on June 20, 1990. She received the B.E. degree in electronically engineering from Shizuoka University in 2013. She has been with the Graduate School of Engineering, Shizuoka University since 2013. Her research interests are in multi-hop networks and error detection and correct code.

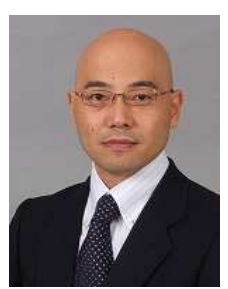

Kouji Ohuchi received the B.E., M.E., and Eng.D degrees from Ibaraki University, in 1994, 1996, and 1999, respectively. He joined in Graduate School of Electronic Science and Technology, Shizuoka University in 1999 as a research associate. He has been an associate professor at Faculty of Engineering, Shizuoka University since 2007 . He is a member of IEEE. His research interests are in spread-spectrum communication systems, multi-carrier transmission techniques, and multi-hop networks. 